\title{
Airline Schedule Competition: Product-Quality Choice in a Duopoly Model
}

\author{
by \\ Jan K. Brueckner \\ Department of Economics \\ University of California, Irvine \\ 3151 Social Science Plaza \\ Irvine, CA 92697 \\ e-mail: jkbrueck@uci.edu \\ and \\ Ricardo Flores-Fillol \\ Departament d'Economia i d'Història Econòmica \\ Universitat Autònoma de Barcelona \\ Edifici $B$ \\ Bellaterra (Barcelona) 08193, Spain \\ e-mail: rflores@idea.uab.es
}

April 2006

\begin{abstract}
This paper presents a simple model of airline schedule competition that circumvents the complexities of the spatial approach used in earlier papers. Consumers choose between two duopoly carriers, each of which has evenly spaced flights, by comparing the combinations of fare and expected schedule delay that they offer. In contrast to the spatial approach, the particular departure times of individual flights are thus not relevant. The model generates a number of useful comparative-static predictions, while welfare analysis shows that equilibrium flight frequencies tend to be inefficiently low.
\end{abstract}




\title{
Airline Schedule Competition: Product-Quality Choice in a Duopoly Model
}

by

\author{
Jan K. Brueckner and Ricardo Flores-Fillol*
}

\section{Introduction}

Despite the existence of a large theoretical literature analyzing the economics of airline behavior, little attention has been devoted to a key aspect of airline operations: scheduling decisions in competitive markets. Since the convenience of airline schedules is a paramount concern of passengers along with the fares they pay, this omission represents a serious gap in

the theoretical literature. ${ }^{1}$ The purpose of the present paper is to help fill this gap by analyzing a simple model of schedule competition in a duopolistic airline market.

The few existing papers on schedule competition mostly take a spatial approach to the problem. In these models, consumers have a distribution of desired departure times, and airlines set their flight schedules taking account of this distribution, along with the schedules of competitors, while also setting fares. In its most general form, the resulting problem is one of spatial competition, where a long tradition of analysis exists. d'Aspremont et al. (1979) present the classic treatment of the simplest spatial-competition problem, where two firms each choose a single location (flight time) along a line while also setting prices. While this problem is difficult enough, the most general analysis of schedule competition would allow firms to endogenously choose, in Nash fashion, the number of locations at which they operate (number of flights), a choice that requires prior computation of the spatial-competition equilibrium for each possible combination of flight numbers for the two firms.

Because this problem is unmanageable analytically, various simplifying assumptions are imposed in the papers that analyze spatial schedule competition. Schipper et al. (2003, 2006) suppress the locational-choice dimension of the problem by assuming that flights are spaced equally around a circle, with competitors' flights interleaved to the maximum extent possible. In the duopoly case, interleaving is perfect when flight numbers are equal (with adjacent flights 
always belonging to different airlines), but otherwise, some adjacent flights belong to the same firm. By contrast, Lindsey and Tomaszewska's (1998) analysis eliminates the choice of flight frequency, with each airline operating a fixed number of flights. Once a consumer has identified each airline's best flight from among those it operates, the remaining choice between airlines is governed by a random utility model. The analysis of the resulting flight-location/fare equilibria is done numerically. ${ }^{2}$

To avoid the complexities of spatial models, the present paper takes a non-spatial approach to analyzing schedule competition. The analysis uses elements of the monopoly scheduling model of Brueckner (2004) along with the brand-loyalty framework from Brueckner and Whalen (2000). In Brueckner (2004), consumers must commit to travel before knowing their preferred departures times, which are uniformly distributed around a circle. Letting $T$ denote the time circumference of the circle, consumer utility then depends on expected schedule delay, ${ }^{3}$ which equals $T / 4 f$, where $f$ is number of (evenly spaced) flights operated by the monopoly airline. Adapting this approach to a competitive setting, the consumer is assumed to compare expected schedule delays for the two duopoly airlines, $T / 4 f_{1}$ and $T / 4 f_{2}$, along with the fares they charge, $p_{1}$ and $p_{2}$, in making a choice between them. While this approach may not be fully accurate for individual consumers, it appears to capture the choice setting of a corporate travel department, which must sign an exclusive contract with a particular airline for transporting its employees. The travel department cares about the average schedule delay for the company employees, while also seeking low fares. It signs an exclusive contract with the airline providing the best combination of these features. Alternatively, the model could apply to individual business travelers, who cannot predict their travel times and thus purchase refundable full-fare tickets, which allow them to board the next flight upon arriving at the airport. In either case, the precise departure times of individual flights are not relevant, accounting for the simplicity of the overall approach.

Without further assumptions, the airline with the most attractive frequency/fare combination would attract all the passengers in the market. To avoid this outcome, consumers (corporate travel departments) are presumed to exhibit brand loyalty to particular carriers, which means that an airline with an inferior frequency/fare combination can still attract some 
passengers. Following Brueckner and Whalen (2000), this approach is formalized by specifying a utility gain from using airline 1 rather than airline 2, denoted $a$, and assuming that this gain is uniformly distributed over the range $[-\alpha / 2, \alpha / 2]$, so that half the consumers prefer airline 1 and half prefer airline 2. Note that $\alpha$ is a measure of (exogenous) product differentiation in the sense that a small $\alpha$ implies a small maximum gain from using one airline or the other, indicating similar products. ${ }^{4}$

Under the above assumptions, the two carriers compete by choosing both frequencies and fares to maximize profit. The resulting model is very similar in its broad outlines to the framework used by Heimer and Shy (2006) to study the formation of airline alliances, which they portray as benefiting passengers via the higher flight frequencies made possible by combining airline operations. The present model differs, however, by using more-realistic functional forms and by offering a richer specification of the market setting. These differences will be spelled out more fully in the ensuing discussion.

The model is also related to the literature on vertical product differentiation, initiated by Gabszewicz and Thisse (1979) and Shaked and Sutton (1982) and summarized by Tirole (1988). ${ }^{5}$ In such models, consumer utility is usually given by $\lambda s-p$, where $s$ is product quality and $p$ is price. As seen below, the analogous portion of the utility function in the present model is $-\gamma / f-p$, which differs only in the reciprocal form of the quality measure (flight frequency). Despite this similarity, the sources of consumer heterogeneity are entirely different in the two setups. While consumers have different valuations of product quality $(\lambda)$ in vertical product-differentiation models, all consumers value flight frequency equally under the present approach. Heterogeneity arises instead through brand loyalty, which introduces a consumer-specific additive shift factor (rather than slope variation) into the utility function. As noted above, the dispersion of this brand-loyalty factor, which ranges over $[-\alpha / 2, \alpha / 2]$, captures an exogenous degree of product differentiation that is built into the model rather than determined in equilibrium.

Because of this difference in the treatment of consumer heterogeneity, equilibria differ in a fundamental way under the two approaches. In the vertical-differentiation literature, endogenous product differentiation emerges, with one firm choosing the highest possible product 
quality and the other choosing a lower quality, as firms seek to soften price competition. Consumers select between the two goods according to their valuations of quality. With all consumers valuing flight frequency equally in the present model, an impetus for the delivery of different product qualities does not exist. The relevant equilibrium is then symmetric, with both firms offering identical frequencies, while recognizing that their quality choices affect demand. Note that a symmetric equilibrium is realistic in the airline context, where competing firms usually offer similar flight frequencies. Observe also that, despite its portrayal of flightfrequency competition, a model of this type could be applied more generally in other contexts within industrial organization.

The goal of the paper is to demonstrate the tractability and usefulness of the non-spatial approach to schedule competition, while also highlighting the general value of this alternative portrayal of product-quality competition. This goal is achieved by exploring the nature and comparative-static properties of equilibrium and by carrying out welfare analysis. The plan of the paper is as follows. Section 2 analyzes the model under the assumption that fares and flight frequencies are chosen simultaneously by the duopolists. Comparative-static analysis of the resulting equilibrium is presented, showing how equilibrium fares and frequencies depend on the various parameters of the model. Because some of the comparative-static effects are ambiguous in sign, section 3 presents illustrative numerical examples. Section 4 characterizes the social optimum, comparing optimal frequencies and traffic levels to those emerging in equilibrium. Section 5 considers sequential (rather than simultaneous) choices of fares and frequencies, with the frequency choice made first, and compares the resulting equilibrium to both the simultaneous-choice equilibrium and the social optimum. Section 6 offers conclusions.

\section{The Model and the Simultaneous-Choice Equilibrium}

\subsection{Basic analysis}

In the model, utility for a consumer traveling by air is given by $c+$ travel benefit schedule delay cost, where $c$ is consumption expenditure. Schedule delay cost is equal to a disutility parameter $\delta>0$ times the expected schedule delay expression from above, thus equaling $\delta T / 4 f_{i} \equiv \gamma / f_{i}$ for airline $i, i=1,2$, where $\gamma=\delta T / 4$. Travel benefit has two 
components: $b$, equal to the gain from air travel, and $a$, the airline brand-loyalty variable from above, which gives the additional gain from using airline 1 (relative to travel on airline 2). Recall that $a$ varies across consumers. For consumers using airline $i$, consumption expenditure equals $y-p_{i}$, where $y$ is income, assumed to be uniform across consumers without loss of generality, and $p_{i}$ is airline $i$ 's fare. Utility from travel on airline 1 is thus given by $y-p_{1}+b+$ $a-\gamma / f_{1}$, and utility from travel on airline 2 is given by the analogous expression with $a=0 .{ }^{6}$

Assuming that the consumer undertakes air travel, he will choose airline 1 when

$$
y-p_{1}+b+a-\gamma / f_{1}>y-p_{2}+b-\gamma / f_{2}
$$

or when

$$
a>p_{1}-p_{2}+\gamma / f_{1}-\gamma / f_{2}
$$

Thus, for the consumer to choose airline 1, brand loyalty to 1 must be at least as large as the fare plus delay-cost difference between airlines 1 and 2. Otherwise, conditional on flying, the consumer will choose airline 2. As for the air-travel decision itself, the benefit from the consumer's "outside option" (the next best alternative) must be considered. That option might involve not traveling at all, but more realistically, the outside option could be travel using a different transport mode, such as automobile or train. Whatever its characteristics, the outside option will be unattractive when the air-travel benefit $b$ is sufficiently high, making the margin of choice the one between airlines 1 and 2. One group of consumers in the model satisfies this requirement, having a common high level of the air-travel benefit, denoted $b^{H}$. This high-benefit group (denoted type $H$ ) represents a proportion $\mu$ of the consumer population.

To use (2) to compute airline 1's passenger traffic from among the type- $H$ group, let the consumer population size be normalized to unity, and recall that $a$ is uniformly distributed over the range $[-\alpha / 2, \alpha / 2]$. Then airline 1 's type- $H$ traffic is given by

$$
q_{1}^{H}=\mu \int_{p_{1}-p_{2}+\gamma / f_{1}-\gamma / f_{2}}^{\alpha / 2} \frac{1}{\alpha} d a
$$

where $1 / \alpha$ gives the density of $a$. 
The rest of the population, representing a fraction $1-\mu$, has a common low level of the air-travel benefit, denoted $b^{L}$. It is assumed that, in equilibrium, a portion of this group ends up not undertaking air travel, an outcome that is assured when $b^{L}$ and other parameters in the model take appropriate values (see below). To understand this outcome, let the common benefit level from the outside option, net of any fare paid or schedule delay incurred, be denoted $g$, yielding a utility level of $y+g$. Then consider a consumer who is indifferent between the airlines, with $a=0$. This individual will prefer the outside option to traveling on airline 1 if $y-p_{1}+b^{L}-\gamma / f_{1}<y+g$, or if $b^{L}-g<p_{1}+\gamma / f_{1}$, and will prefer the outside option to traveling on airline 2 if $b^{L}-g<p_{2}+\gamma / f_{2}$. If both inequalities are satisfied, a type- $L$ consumer indifferent between the airlines thus will not undertake air travel. But the additional benefit from airline brand loyalty may tip the balance if it is strong enough. For example, a passenger loyal to airline 1 will find air travel and the outside option equally attractive when his loyalty value satisfies $y-p_{1}+b^{L}+a-\gamma / f_{1}=y+g$, or $a=p_{1}+\gamma / f_{1}-b^{L}+g \cdot{ }^{7}$ Normalizing $g$ to zero for simplicity, consumers with $a$ values larger than

$$
\widehat{a}=p_{1}+\gamma / f_{1}-b^{L}
$$

will then strictly prefer travel on airline 1 to the outside option. Since $b^{L}<p_{2}+\gamma / f_{2}$ holds by assumption, the inequality in (2) is satisfied for all these passengers, indicating that airline 1 is also preferred to airline 2. Therefore, airline 1's passenger traffic from among the type- $L$ group is equal to

$$
q_{1}^{L}=(1-\mu) \int_{\widehat{a}}^{\alpha / 2} \frac{1}{\alpha} d a .
$$

The margin of choice for the type- $L$ group is thus between the preferred airline and the outside option, while the type- $H$ choice margin is between airlines 1 and 2 . Observe, however, that the relevant margin for a given group is in fact endogenous and dependent on equilibrium fares and frequencies. ${ }^{8}$ It is assumed that, in equilibrium, the relevant margins are as indicated.

Carrying out the integration in (3) and (5) and adding the results, airline 1's total traffic, 
$q_{1}^{H}+q_{1}^{L}$, is equal to

$$
q_{1}=\frac{1}{2}-\frac{1}{\alpha}\left[p_{1}-\mu p_{2}+\gamma / f_{1}-\mu \gamma / f_{2}-(1-\mu) b^{L}\right]
$$

and airline 2's traffic is given by the analogous expression with the 1 and 2 subscripts interchanged. Note from (6) that airline 1 loses traffic when its fare rises or its frequency falls, while it gains traffic when $p_{2}$ rises or $f_{2}$ falls. In the first case, type- $H$ traffic is lost to airline 2 , and type- $L$ traffic is lost to the outside option. To further interpret (6), suppose that the entire population is of type $H$, so that $\mu=1$. Then, in a symmetric equilibrium, where $p_{1}=p_{2}$ and $f_{1}=f_{2},(6)$ indicates that the airlines each carry traffic of $1 / 2$, equal to half of the total unitary population (all of which undertakes air travel). When $\mu<1$, traffic is less than $1 / 2$, but its magnitude depends on the equilibrium fare and frequency.

To characterize the equilibrium, the airline cost structure, which is symmetric across the carriers, must be specified. Following Brueckner (2004), an airline operates aircraft with $s$ seats, with the load factor assumed to equal 100\%, so that all seats are filled. A flight's operating cost is given by $\theta+\tau s$, where $\theta$ is a fixed cost independent of aircraft size and $\tau$ is the marginal cost per seat. ${ }^{9}$ Under this specification, cost per seat realistically falls with aircraft size. Flight frequency, $s$ and traffic are all related by the equation $q=f s$, which says that an airline's total traffic equals frequency times seats per flight. Thus, while $s$ is endogenous, its value is determined residually once $q$ and $f$ are known. Viewing $f$ as product quality, this cost structure presumes that quality has a fixed cost but no associated variable cost, an assumption that is sometimes used in the vertical product-differentiation literature.

Using the above information and (6), airline 1's profit is equal to

$$
\begin{gathered}
\pi_{1}=p_{1} q_{1}-f_{1}\left(\theta+\tau s_{1}\right) \\
=p_{1} q_{1}-\theta f_{1}-\tau q_{1} \\
=\left(p_{1}-\tau\right)\left(\frac{1}{2}-\frac{1}{\alpha}\left[p_{1}-\mu p_{2}+\gamma / f_{1}-\mu \gamma / f_{2}-(1-\mu) b^{L}\right]\right)-\theta f_{1} .
\end{gathered}
$$

Note in the second line of (7) that costs separate into the fixed cost of flights and the variable cost of seats, which is independent of the number of flights. 
Given that an airline can adjust its flight frequency fairly easily, it may be reasonable to assume that, in maximizing profit, fares and frequencies are chosen simultaneously. For comparison purposes, the sequential case (where frequency is chosen first) is considered below. With simultaneous choice, airline 1 chooses $p_{1}$ and $f_{1}$ to maximize $(7)$, viewing $p_{2}$ and $f_{2}$ as parametric. The first-order conditions are

$$
\begin{gathered}
\frac{\partial \pi_{1}}{\partial p_{1}}=\frac{1}{2}-\frac{1}{\alpha}\left[p_{1}-\mu p_{2}+\gamma / f_{1}-\mu \gamma / f_{2}-(1-\mu) b^{L}\right]-\frac{p_{1}-\tau}{\alpha}=0 \\
\frac{\partial \pi_{1}}{\partial f_{1}}=\left(p_{1}-\tau\right) \frac{\gamma}{\alpha f_{1}^{2}}-\theta=0 .
\end{gathered}
$$

The second-order conditions $\partial^{2} \pi_{1} / \partial p_{1}^{2}, \partial^{2} \pi_{1} / \partial f_{1}^{2}<0$ are satisfied by inspection, and the remaining positivity condition on the Hessian determinant is assumed to hold.

With firm symmetry, the symmetric equilibrium is the natural focus, and this equilibrium is found by setting $p_{1}=p_{2}=p, f_{1}=f_{2}=f$ in (8) and (9) and solving for these common values. Substituting (9) into (8) and rearranging, the $f$ solution satisfies

$$
\left[\alpha \gamma / 2+(1-\mu) \gamma\left(b^{L}-\tau\right)\right] f-(1-\mu) \gamma^{2}=(2-\mu) \alpha \theta f^{3}
$$

and the $p$ solution conditional on $f$ is then found by rearranging (9):

$$
p=\frac{\alpha \theta f^{2}}{\gamma}+\tau \text {. }
$$

The $f$ solution in (10) occurs at an intersection of the cubic curve represented by the RHS and the line represented by the LHS, whose vertical intercept is negative. If $f$ 's coefficient on the LHS is also negative, then (10) has no positive solution (the LHS is then negative for any positive $f$ ). As a result, the $f$ coefficient must be positive, giving the RHS line a positive slope. The $f$ solution is then given by one of the intersections in the positive quadrant, as seen in Figure 1, and it is easily seen that only the second of these intersections satisfies the second-order condition. ${ }^{10}$ 


\subsection{Comparative statics}

To illustrate the properties of the equilibrium, this section presents comparative-static analysis. To start, it is interesting to consider the solution that emerges when $\mu=1$, indicating the absence of type- $L$ consumers. Substituting $\mu=1$ into (10) and (11) yields

$$
\begin{aligned}
& f=\sqrt{\gamma / 2 \theta} \\
& p=\tau+\alpha / 2 .
\end{aligned}
$$

Thus, the fare $p$ equals the marginal cost of a seat $(\tau)$ plus a markup that depends on the degree of product differentiation $(\alpha / 2)$. As differentiation disappears (with $\alpha$ converging to zero), the fare converges to marginal cost, the Bertrand-equilibrium outcome. Frequency $f$, on the other hand, is independent of $\tau$ and $\alpha$ but rises with the disutility of schedule delay $(\gamma)$ and falls as the fixed flight cost $\theta$ rises, both intuitive conclusions. Note that since the entire population undertakes air travel in the absence of type- $L$ consumers, traffic remains fixed at $1 / 2$ regardless of parameter values. ${ }^{11}$

To conduct comparative-static analysis when $\mu<1$, it is useful to rewrite (8) in the following two different forms, after imposing symmetry:

$$
p=\frac{\alpha / 2-(1-\mu)\left(\gamma / f-b^{L}\right)+\tau}{2-\mu} ; \quad q=\frac{p-\tau}{\alpha}
$$

Compared to (11), the first equation in (14) provides an alternate solution for $p$ in terms of $f$ that is useful in evaluating comparative-static effects. Once the effect of a parameter change on $p$ is known, the second equation in (14) is used to determine the impact on traffic. The analysis can be carried out mostly by inspection, using (14) along with (10), (11) and Figure 1.

An increase in the fixed flight $\operatorname{cost} \theta$ raises the height of the cubic curve in Figure 1's positive quadrant without affecting the line, leading to a decrease in $f$. While the $p$ effect from (11) appears ambiguous, (14) shows that $p$ actually falls, as does $q$. Since costs have risen, this fare decrease is counterintuitive, but the apparent explanation comes from the decline in 
frequency, which worsens service quality (the drop in traffic in response to higher cost does make sense, however). Note the contrast to the $\mu=1$ case, where $p$ is unaffected by the level of fixed cost. Thus, for $\theta$ to affect fares, an air-travel/outside-option margin must exist, so that total traffic is endogenous rather than fixed at a value of $1 / 2$.

When the marginal seat cost $\tau$ rises, the slope of the line in Figure 1 falls, reducing $f$. However, the changes in both $p$ and $q$ are ambiguous. Recall, by contrast, that a higher $\tau$ leaves $f$ unchanged while unambiguously raising $p$ in the $\mu=1$ case.

When the disutility of schedule delay $(\gamma)$ rises, the slope of the line changes in an ambiguous direction while its intercept becomes more negative, leading to an ambiguous change in $f$. However, as $\mu$ approaches 1 , the intercept approaches zero and the effect of its change becomes negligible, while the slope impact becomes unambiguously positive. The positive slope effect thus dominates the outcome, raising $f$. While this impact matches intuition, the associated changes in $p$ and $q$ are ambiguous.

When product differentiation $(\alpha)$ increases, both the slope of the line and the height of the cubic curve rise, again suggesting an ambiguous effect on $f$. However when the type- $L$ travel benefit is low, with $\tau>b^{L}$ holding, the net effect is to push the intersection to the right, raising $f .^{12}$ From (11) or (14), $p$ rises, while the change in $q$ from (14) is ambiguous. Thus, when the perceived brand difference between the two airlines widens, flight frequency and the fare rise provided that $b^{L}$ is less than the marginal cost of a seat, although traffic could rise or fall.

Given that greater product differentiation should weaken price competition between the carriers, the emergence of a higher fare makes sense. While this logic also explains the rise in $p$ when $\mu=1$, flight frequency is unaffected by the extent of product differentiation in this case. The difference is again explained by the existence of an air-travel/outside-option margin when $\mu<1$. In this case, a higher $\alpha$, by raising the upper limit of integration in (5), initially increases the number of type- $L$ consumers choosing air travel. This fact then induces adjustments in $f$ and $q$, although the ultimate change in traffic is ambiguous.

When $b^{L}$ rises, the slope of the line in Figure 1 increases, raising $f$. Using (11) or (14), $p$ then rises, as does $q$ from (14). Since the increase in $b^{L}$ raises the demand for air travel among 
type- $L$ consumers, these responses also make sense.

Finally, when $\mu$ increases, the cubic curve shifts down, while the line's intercept shifts up toward zero. If $\tau>b^{L}$, then the line's slope rises, leading to an increase in $f$. In this case, (11) and (14) show that both $p$ and $q$ also rise. Thus, if the air-travel benefit for type- $L$ consumers is low, a decline in their population share raises frequency, the fare and traffic. Otherwise, the effects are ambiguous.

These results are summarized as follows, and in Table 1 (which also shows results for the $\mu=1$ case):

Proposition 1. If the population includes some type- $L$ consumers $(\mu<1)$, then:

(i) Flight frequency falls with an increase in the fixed flight cost or marginal seat cost. Frequency rises with an increase in the disutility of schedule delay (when $\mu$ is sufficiently large), product differentiation (when $\tau>b^{L}$ ), the type-L air-travel benefit, or the share of type- $H$ passengers (when $\tau>b^{L}$ ).

(ii) The fare falls with an increase in the fixed flight cost. The fare rises with an increase in product differentiation (when $\tau>b^{L}$ ), the type-L air-travel benefit, or the type-H share (when $\left.\tau>b^{L}\right)$.

(iii) Traffic falls with an increase in the fixed flight cost. Traffic rises with an increase in the type-L air-travel benefit or the type-H share $\left(\right.$ when $\left.\tau>b^{L}\right)$.

To provide a sense of the likely directions of those comparative-static effects that are theoretically ambiguous, the next section offers some numerical examples.

\section{Numerical Examples}

Given the stylized nature of the model, parameter choices are necessarily arbitrary. The requirement $\tau>b^{L}$ from above is imposed, however, and parameters are chosen so that the critical value of brand loyalty $\widehat{a}$ above which type- $L$ consumers undertake air travel is less than $\alpha / 2$, making this set of consumers nonempty (see (5)). ${ }^{13}$ Given these requirements, the base-case parameter values are $\alpha=30, b^{L}=18, \gamma=0.2, \theta=25, \tau=20$, and $\mu=0.5$. Note the type- $L$ air-travel benefit is only slightly greater than the maximum brand-loyalty benefit (equal to 15 ). Since $b^{L}$ is measured relative to the benefit $g$ from the outside option, which is normalized to zero, this small gap is plausible. In addition, the fixed $\operatorname{cost} \theta$ is of the same 
order of magnitude as the marginal seat $\operatorname{cost} \tau$, indicating weak increasing returns, and the type- $L$ and type- $H$ groups are equal in size. Note finally that the schedule-delay disutility parameter $\gamma$ has no natural connection to any of the other parameter values and can be set independently.

Recall that the variable $s$, seats per flight, is determined residually via choice of $f$ and $p$ (which together determine $q$ and hence $s=q / f$ ). Since the comparative statics for $s$ are all analytically ambiguous, numerical results for this variable are presented along with results for the variables $f, p, q$ and $\pi$ (profit). The first line of Table 2 shows the base case solution. While the magnitudes of the solution values are not particularly meaningful, the remainder of the Table shows how these values change when parameters are altered.

When the fixed cost $\theta$ increases from 25 to $26, f, p$ and $q$ all decline, as in Proposition 1. In addition, Table 2 shows that the decline in $q$ is proportionally smaller than the decline in $f$, so that $s=q / f$ rises, indicating use of larger aircraft. This outcome makes intuitive sense given the higher fixed cost per flight.

When the marginal cost per seat rises from 20 to 21, $f$ falls, matching the prediction of Proposition 1. Although the general effects are ambiguous, the fare rises and traffic falls in the example, an outcome always observed in the computations. Note that, unlike in case of a fixed-cost increase, which counterintuitively lowers the fare, the positive effect of a higher $\tau$ on $p$ matches intuition. In addition, aircraft size falls, again an intuitive outcome.

When the schedule-delay disutility $\gamma$ increases from 0.2 to 0.3 , frequency rises. While Proposition 1 predicts this result when $\mu$ is sufficiently large, the assumed value of 0.5 is evidently large enough. In addition, Table 2 shows that $\gamma$ 's impacts on the fare and traffic, ambiguous in general, are both negative in the example, an outcome that emerged in all of the computations. Aircraft size also falls. Thus, the results suggest that a greater dislike of schedule delay leads to more-frequent, less-expensive flights on smaller aircraft.

When product differentiation rises (with $\alpha$ increasing from 30 to 31), all the solution values increase. The higher values of $f, p$, and $q$ are consistent with Proposition 1 given $\tau>b^{L}$. Since the increase in traffic is proportionally greater than the increase in frequency, $s$ rises, so that larger aircraft are used. 
When the type- $L$ air-travel benefit $b^{L}$ rises from 18 to 19 , all the solution values increase, results that are again consistent with Proposition 1. Aircraft size $s$ again rises, a conclusion consistent with the demand increase reflected in the higher $b^{L}$.

Finally, when the type- $H$ share rises from 0.5 to 0.55 , all the solution values increase, an outcome consistent with Proposition 1 given $\tau>b^{L}$. Note that the increase in aircraft size again reflects the demand increase associated with a higher $\mu$.

As for the profit changes, all are consistent with predicted effects, which can be computed as partial derivatives of the $\pi$ function in (7), using the envelope theorem.

\section{The Social Optimum}

With the comparative-static properties of the equilibrium understood, attention now shifts to welfare analysis, where a social planner chooses flight frequency and traffic. Social surplus is computed as the sum of total utility and airline profit. Letting $a^{*}$ denote the air-travel/outsideoption loyalty margin for type- $L$ consumers, as chosen by the planner, total utility can be written

$$
\begin{aligned}
U=2 \mu & \int_{0}^{\alpha / 2}\left(y-p+b^{H}+a-\gamma / f\right) \frac{1}{\alpha} d a \\
& +2(1-\mu)\left[\int_{a^{*}}^{\alpha / 2}\left(y-p+b^{L}+a-\gamma / f\right) \frac{1}{\alpha} d a+\frac{a^{*} y}{\alpha}\right] .
\end{aligned}
$$

Note that the 2 factors in (15) are needed because two airlines are present, and that the last term inside the brackets captures utility for type- $L$ consumers choosing the outside option.

Total airline profit is given by

$$
2 \pi=2\left((p-\tau)\left[\mu \frac{1}{2}+(1-\mu)\left(\frac{1}{2}-\frac{a^{*}}{\alpha}\right)\right]-\theta f\right)
$$

where $(1-\mu)\left(1 / 2-a^{*} / \alpha\right)$ gives the number of type- $L$ passengers per airline. Carrying out the integration in (15) and adding (16), surplus is equal to

$$
W=y+\mu \frac{\alpha}{4}+(1-\mu)\left[\frac{\alpha}{4}-\frac{a^{* 2}}{\alpha}\right]+\mu b^{H}+(1-\mu)\left[1-\frac{a^{*}}{\alpha / 2}\right] b^{L}
$$




$$
-(\tau+\gamma / f)\left(\mu+(1-\mu)\left[1-\frac{a^{*}}{\alpha / 2}\right]\right)-2 \theta f
$$

Note that $b^{L}$ 's coefficient equals the total number of type- $L$ passengers, so that the last two terms in the first line of (17) give aggregate air-travel benefits. The last line gives flight costs plus the cost of schedule delay, and the second term in the first line gives total brand-loyalty benefits for type- $H$ passengers (their population share times average benefits of $\alpha / 4$ ). The third term gives the same quantity for type- $L$ passengers. ${ }^{14}$

The first-order condition for choice of $a^{*}$ reduces to

$$
a^{*}=\tau+\gamma / f-b^{L},
$$

indicating that, for the marginal type- $L$ air traveler, the benefits from air travel and brand loyalty are exactly balanced by the marginal seat cost seat plus the disutility from schedule delay. The condition for choice of $f$ is $2 \theta-\left(\gamma / f^{2}\right)\left[1-(1-\mu) 2 a^{*} / \alpha\right]=0$, and substituting (18) and rearranging, it reduces to

$$
\left[\alpha \gamma / 2+(1-\mu) \gamma\left(b^{L}-\tau\right)\right] f-(1-\mu) \gamma^{2}=\alpha \theta f^{3} .
$$

Using these conditions, the social optimum and equilibrium are easily compared. Note first that (19) is identical to the equilibrium condition (10) except for the absence of the $2-\mu$ coefficient multiplying $f^{3}$. Thus, the cubic curve in (19) is lower than the cubic curve in the equilibrium condition as long as $\mu<1$, while the curves coincide when $\mu=1$. As a result, the socially optimal flight frequency is higher than the equilibrium frequency as long the population contains some type- $L$ consumers. If everyone is of type- $H$, the equilibrium frequency is optimal, with both equaling $\sqrt{\gamma / 2 \theta}$.

In addition, when $\mu<1$, the equilibrium number of type- $L$ consumers undertaking air travel is too small. To see this conclusion, recall from (4) that the equilibrium airtravel/outside-option margin for type- $L$ consumers equals $\widehat{a}=p+\gamma / f-b^{L}$. Since $p>\tau$ from (11) and since the equilibrium frequency is too low, it follows from (18) that $a^{*}<\widehat{a}$. 
Thus, at the optimum, an additional group of type- $L$ consumers with relatively low brand loyalties ends up undertaking air travel. Note that when $\mu=1$, the entire population undertakes air travel both at the optimum and in equilibrium, so that no consumers are inefficiently excluded.

Summarizing these results yields

Proposition 2. If the population includes some type-L consumers, then the equilibrium has inefficiently low flight frequency and too few type-L air travelers. If the entire population is of type $H$, then the equilibrium is efficient.

As was shown in the analysis of section 2, the comparative-static properties of the equilibrium differ markedly between the $\mu=1$ and $\mu<1$ cases. Proposition 2 shows that the same conclusion applies to its efficiency properties. The proposition shows that equilibrium is inefficient only in the case where airline market power affects total traffic (i.e., when an airtravel/outside-option margin exists). In this case, each airline has effective monopoly power over its type- $L$ passengers, whose next best choice is the outside option. In a familiar fashion, this monopoly power leads to a suboptimal level of traffic and a corresponding underprovision of frequency. By contrast, when $\mu=1$, the exercise of market power only affects the division of a fixed amount of traffic between the carriers, and inefficiency disappears. In this case, airlines exert no monopoly power over any passenger, although they can still affect the division of the fixed traffic pool by their choices of fares and frequencies. This difference between the two cases also accounts for the divergence in comparative-static results between them, as seen in Table 1.

\section{The Sequential-Choice Equilibrium}

While the analysis in section 2 assumed simultaneous choice of fares and frequencies, arguing that both variables are easily adjusted, it could be argued that fares can be adjusted more quickly than frequencies. If this view is appropriate, then a sequential-choice model, where frequency is chosen before fares, may be more realistic. In such a model, fares are chosen conditional on frequencies in a second stage, with frequencies chosen in a first stage taking into account their second-stage fare impacts. The outcome is a subgame perfect Nash 
equilibrium. ${ }^{15}$

To carry out the required analysis, the fare-choice condition (8) for airline 1 is supplemented by the analogous condition for airline 2, and the two equations are solved simultaneously to give $p_{1}$ and $p_{2}$ as functions of $f_{1}$ and $f_{2}$. The solution for $p_{1}$ conditional on $f_{1}$ and $f_{2}$ is

$$
p_{1}=\frac{1}{2-\mu^{2} / 2}\left[(\alpha / 2+\tau)(1+\mu / 2)-\left(1-\mu^{2} / 2\right) \gamma / f_{1}+\mu \gamma / 2 f_{2}+\left(1-\mu / 2-\mu^{2} / 2\right) b^{L}\right]
$$

and the $p_{2}$ solution is gotten by reversing the 1 and 2 subscripts.

These solutions are then substituted into airline 1's profit function, which becomes a function of just $f_{1}$ and $f_{2}$. The derivative of this function with respect to $f_{1}$ is set equal to zero, and symmetry is then imposed by setting $f_{1}=f_{2}=f$ in the resulting equation. After substantial manipulation, the following equilibrium condition emerges:

$$
\frac{(\alpha \gamma / 2)(2+\mu)+\left(2-\mu-\mu^{2}\right) \gamma\left(b^{L}-\tau\right)}{2-\mu^{2} / 2} f-\frac{2-\mu-\mu^{2}}{2-\mu^{2} / 2} \gamma^{2}=\frac{\left(2-\mu^{2} / 2\right)}{1-\mu^{2} / 2} \alpha \theta f^{3} .
$$

Note that this equation has the same general form as (10).

The comparative-static properties of the sequential equilibrium are summarized as follows:

Proposition 3. When $\mu<1$, the comparative-static effects of $\theta, \tau, \gamma, \alpha$ and $b^{L}$ on $f, p$, and $q$ are the same as in Proposition 2. The effect of an increase in $\mu$, however, is ambiguous.

This proposition follows from repetition of the previous arguments. The $\mu$ effect is ambiguous because a higher $\mu$ raises the height of the cubic curve, moves the line's intercept closer to zero, and has an ambiguous effect on its slope.

As in the simultaneous-choice case, a simple solution emerges when $\mu=1$. In this case, (21) yields

$$
f=\sqrt{\gamma / 3 \theta}
$$

and substituting in (11), the equilibrium fare is given by

$$
p=\tau+\alpha / 3
$$


Note that frequency is smaller than in the simultaneous-choice case (compare (12)), and that the fare involves a smaller markup over marginal seat cost (compare (13)).

While the sequential frequency will remain smaller, by continuity, when $\mu$ is close to 1 , this relationship cannot be established for arbitrary $\mu>0$. However, since the sequential and simultaneous $f$ 's are identical for $\mu=0,{ }^{16}$ it seems likely that the sequential $f$ never rises above the simultaneous value, a conclusion that is confirmed in all of the computations.

Rather than being socially optimal, as before, the equilibrium frequency is too small in the sequential case when $\mu=1(\sqrt{\gamma / 3 \theta}$ vs. $\sqrt{\gamma / 2 \theta})$. Underprovision of frequency also occurs when $\mu<1$ provided that the sequential case has the smaller equilibrium $f$. Since the simultaneouschoice $f$ is then larger as well as inefficiently low by Proposition 2, the result follows. Note that underprovision of frequency, if it occurs, would also be accompanied by inefficiently low traffic in the sequential case, given that $a^{*}<\widehat{a}$ would continue to hold in the sequential equilibrium.

Summarizing the preceding discussion yields

Proposition 4. When frequencies and fares are chosen sequentially and $\mu=1$, the equilibrium frequency is smaller than in the simultaneous-choice case, and numerical results suggest that this conclusion is likely to hold when $0<\mu<1$. Under these circumstances, the sequential-choice frequency is inefficiently low, and traffic is too small when $\mu<1$.

\section{Conclusion}

This paper has presented a simple model of airline schedule competition that circumvents the complexities of the spatial approach used in earlier papers. The framework also has general applicability outside the airline context, offering a different approach to product-quality competition than the usual vertical-differentiation model. The analysis generates a number of useful comparative-static predictions, while also showing that equilibrium flight frequencies tend to be inefficiently low.

While the paper has been devoted to exploring the properties of the model, the non-spatial approach to schedule competition is ripe for applications to particular issues in the airline industry. As mentioned above, Heimer and Shy (2006) use this type of model to study airline alliances, and other applications might be fruitful. For example, Brueckner and Girvin (2006) 
use a version of the model to study the effect of airport noise regulation on flight frequency and aircraft "quietness" in a competitive setting, and the model could also be used in an analysis of airport congestion pricing.

Although the analysis is carried out in the airline context, the approach applies in other transportation settings where scheduling matters. The model, for example, could apply to the market for intercity bus transportation, or to any type of cargo market, where frequency of service is often a concern of shippers. Finally, variants of the model could be used to analyze quality competition in other industries. 


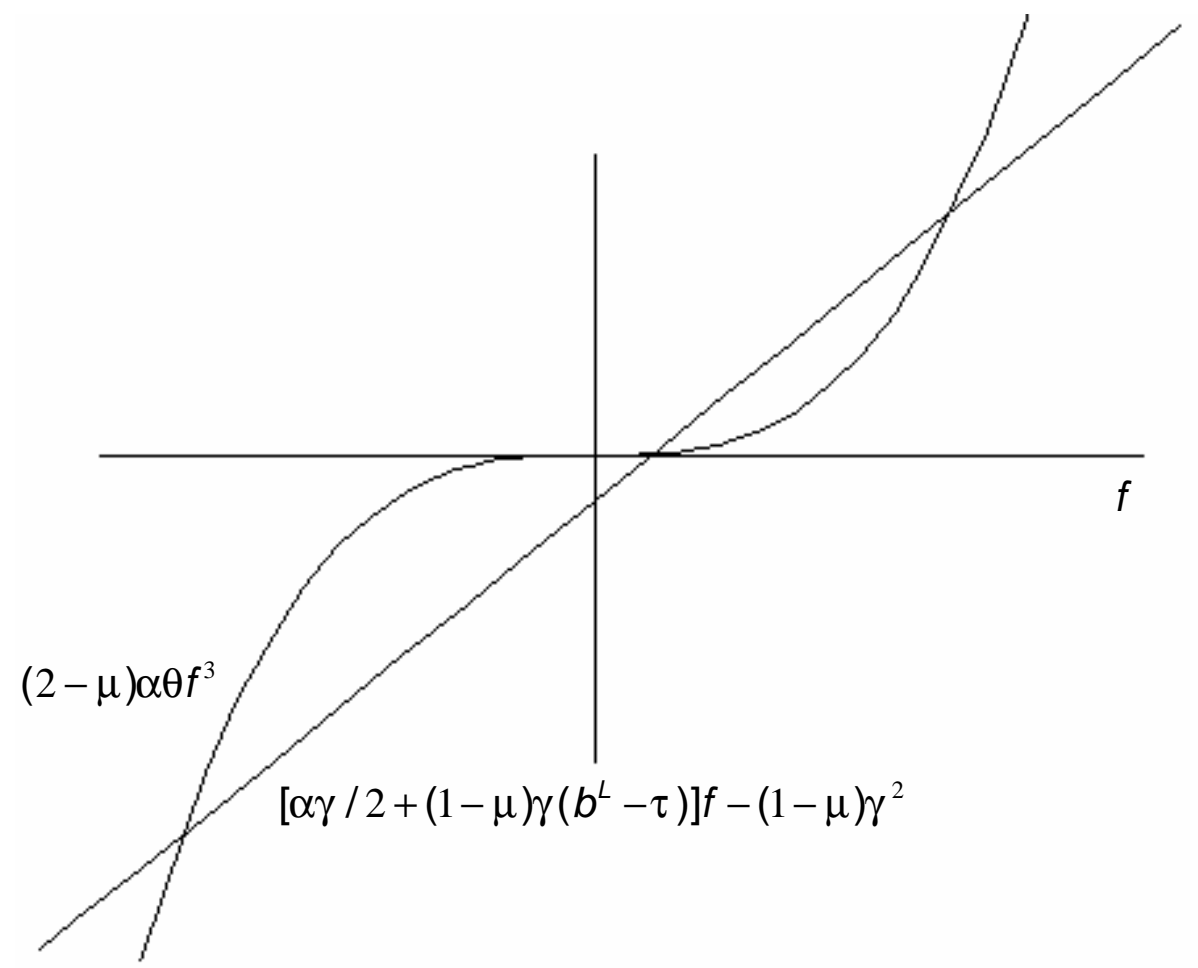

Figure 1: The $f$ solution 
Table 1

Comparative-Static Effects

( $\mu=1$ case in parentheses)

\begin{tabular}{|c|c|c|c|}
\hline \multirow[b]{2}{*}{ Parameter } & \multicolumn{3}{|c|}{ Variable } \\
\hline & $f$ (freq.) & $p$ (fare) & $q$ (traffic) \\
\hline$\theta$ (fixed cost) & $-(-)$ & $-(0)$ & $-(0)$ \\
\hline$\tau($ seat cost $)$ & $-(0)$ & $?(+)$ & $?(0)$ \\
\hline$\gamma(\text { delay disutility })^{*}$ & $+(+)$ & $?(0)$ & $?(0)$ \\
\hline$\alpha$ (product differentiation) $^{* *}$ & $+(0)$ & $+(+)$ & $?(0)$ \\
\hline$b^{L}($ type- $L$ benefit $)$ & $+(0)$ & $+(0)$ & $+(0)$ \\
\hline$\mu(\text { type- } H \text { share })^{* *}$ & $+(0)$ & $+(0)$ & $+(0)$ \\
\hline
\end{tabular}

$* \mu<1$ effects require $\mu$ sufficiently large.

** $\mu<1$ effects require $\tau>b^{L}$. 
Table 2

Numerical Examples for the Simultaneous Case

\begin{tabular}{|c|c|c|c|c|c|}
\hline Cas & $f$ (freq.) & $p$ (fare) & $q$ (traffic) & $s$ (seats) & $\pi$ (profit \\
\hline Base & 0.046 & & & & \\
\hline$\theta \uparrow(\theta=26)$ & $.045(-)$ & 7.847( & $.262(-)$ & .831 & $.886(-)$ \\
\hline$\tau \uparrow(\tau=21)$ & $0.045(-)$ & $28.510(+)$ & $.250(-)$ & $.594(-)$ & $.761(-)$ \\
\hline$\gamma \uparrow(\gamma=0.3)$ & $0.055(+)$ & $09(-)$ & .250 & .567 & $.509(-)$ \\
\hline$\alpha \uparrow(\alpha=31)$ & $0.046(+)$ & $28.219(+)$ & $0.265(+)$ & $5.757(+)$ & $1.028(+)$ \\
\hline$b^{L \uparrow}\left(b^{L}=19\right)$ & $0.047(+)$ & $45(+)$ & $0.275(+)$ & $5.861(+)$ & $.094(+)$ \\
\hline$\mu \uparrow(\mu=0.55)$ & $.047(+)$ & $28.414(+)$ & $0.280(+)$ & $5.921(+)$ & $1.175(+)$ \\
\hline
\end{tabular}

Base case: $\theta=25, \tau=20, \gamma=0.2, \alpha=30, b^{L}=18$ and $\mu=0.5$. 


\section{References}

Brueckner, J.K., 2004. Network structure and airline scheduling, Journal of Industrial Economics 52, 291-312.

Brueckner, J.K., Whalen, T., 2000. The price effects of international airline alliances, Journal of Law and Economics 43, 503-545.

Brueckner, J.K., Zhang, Y., 2001. A model of scheduling in airline networks: How a huband-spoke system affects flight frequency, fares and welfare, Journal of Transport Economics and Policy 35, 195-222.

Brueckner, J.K., GiRvin, R., 2006. Airport noise regulation, airline service quality, and social welfare, unpublished paper, University of California, Irvine.

Choi, C.J., Shin, H.S., 1992. A comment on a model of vertical product differentiation, Journal of Industrial Economics 40, 229-231.

D’Aspremont, C., Gabszewicz, J., Thisse, J.-F., 1979. On Hotelling's stability in competition, Econometrica 47, 1045-1050.

Enchoua, D., Moreaux, M., Perrot, A., 1996. Compatibility and competition in airlines: Demand side network effects, International Journal of Industrial Organization 14, 701-726.

Gabszewicz, J.J., Thisse, J.-F., 1979. Price competition, quality, and income distribution, Journal of Economic Theory 20, 340-359.

Heimer, O., Shy, O., 2006. Code-sharing agreements, frequency of flights, and profits under parallel operation, In: D. Lee (Ed.), Advances in Airline Economics, Vol. 1, Elsevier, Amsterdam.

Lindsey, R., Tomaszewska, E., 1998. Schedule competition, fare competition and predation in a duopoly airline market, unpublished paper, University of Alberta.

Morrison, S.A., Winston, C., 1995. The Evolution of the Airline Industry, Brookings Institution, Washington, D.C.

PANZAR, J.C., 1979. Equilibrium and welfare in unregulated airline markets, American Economic Review 69, 92-95.

SALOP, S.C., 1979. Monopolistic competition with outside goods, Bell Journal of Economics 
$10,141-156$.

Schipper, Y., Nijkamp, P., Rietveld, P., 2003. Airline deregulation and external costs: A welfare analysis, Tranportation Research Part B 37, 699-718.

Schipper, Y., Nijkamp, P., Rietveld, P., 2006. Deregulation and welfare in airline markets: An analysis of frequency equilibria, European Journal of Operations Research, forthcoming.

Shaked, A., Sutton, J., 1982. Relaxing price competition through product differentiation, Review of Economic Studies 49, 3-13.

Tirole, J., 1988. The Theory of Industrial Organization, MIT Press, Cambridge.

Wauthy, X., 1996. Quality choice in models of vertical differentiation, Journal of Industrial Economics 44, 345-353. 


\section{Footnotes}

*Flores-Fillol acknowledges financial support from the Spanish Ministry of Education and Science (fellowship SEC2002-02506 and research grant BEC2003-01132) and Generalitat de Catalunya (research grant 2005SGR00836). We thank Robert Larner for a helpful suggestion, Jiawei Chen and Jun Ishii for guidance on some of the relevant literature, and Robin Lindsey, Inés Macho-Stadler and Xavier Martínez-Giralt for comments.

${ }^{1}$ Estimates by Morrison and Winston (1995) show that the increase in flight frequencies following U.S. airline deregulation has generated cumulative consumer benefits in excess of $\$ 10$ billion, testifying to the importance of airline scheduling decisions.

${ }^{2}$ Using a spatial approach, Brueckner and Zhang (2001) analyze a scheduling model for a monopoly airline, with the goal of understanding how network structure affects flight frequencies. By contrast, Panzar (1979) uses a spatial model to analyze frequency equilibria when each airline operates a single flight. Since free entry along with a zero-profit condition determines equilibrium flight frequency, his model does not depict schedule competition. Salop (1979) presents a similar but more-general model of monopolistic competition in a circular spatial market. Firm locations are interpreted as product brands, but they could also be viewed as flight departure times in the case where each airline offers a single flight. In another analysis of scheduling, Encaoua et al. (1996) analyze the strategic choice of departure times for two airlines when one relies on connecting traffic from the other.

${ }^{3}$ Schedule delay is the difference between the preferred and actual departure times.

${ }^{4}$ The variation in brand loyalty across consumers could be viewed as adding a spatial element to the model, but the key to the analysis is its nonspatial treatment of flight frequencies.

${ }^{5}$ See Choi and Shin (1992) and Wauthy (1996) for more-recent contributions.

${ }^{6}$ Heimer and Shy (2006) assume that consumer utility equals $f-p$ plus a brand-loyalty element that, though different in form, is ultimately equivalent to that in the present specification. While convenient, the linear form of $f$ in their utility function is difficult to justify from first principles.

${ }^{7}$ While $a$ represents the gain from traveling on airline 1 relative to airline 2 , the above condition requires measurement of the absolute gain from traveling on 1. For an easy transition between the relative and absolute cases, it is assumed that when $a>0$, the absolute gain from using airline 1 equals $a$ itself while the absolute gain from airline 2 equals zero. Conversely, 
when $a<0$, the absolute gain from airline 1 equals 0 while the absolute gain from airline 2 equals $a$ itself.

${ }^{8}$ If both $b^{H}$ and $b^{L}$ are sufficiently large, the airline 1-2 margin could be relevant for both the type- $H$ and type- $L$ groups, or the air-travel/outside-option margin could be relevant for both groups if both benefit levels are low. However, the relevant margins for the groups cannot be the reverse of the ones assumed in the text. In contrast to the present setup, Heimer and Shy (2006) assume homogeneous travel benefits and distinguish between two types of equilibria: one where all consumers undertake air travel and the relevant margin is between airlines 1 and 2, and another where the margin is between air travel and the outside option. Although some of the details are different, Brueckner (2004) effectively considers this latter case, where each airline operates as a monopolist.

${ }^{9}$ By contrast, Heimer and Shy (2006) assume that the cost per flight is given by $\theta f+\tau s$, so that fixed cost is increasing in the number of flights, a specification that could be viewed as less natural than the present one.

${ }^{10}$ Alternatively, a single intersection (representing a tangency) might arise, or no intersection might occur in the positive quadrant, in which case a meaningful solution does not exist. To see that the second intersection is relevant when two intersections are present, note that positivity of the Hessian determinant can be shown to require $p-\tau-\gamma / 4 f>0$ or $4 \alpha \theta f^{3}>\gamma^{2}$ using (9) (the firm subscript is dropped). Then observe that, for the second intersection to be relevant, the slope of the cubic curve must exceed the slope of line at the solution, so that $(2-\mu) 3 \alpha \theta f^{3}>\left[\alpha \gamma / 2+(1-\mu) \gamma\left(b^{L}-\tau\right)\right] f$. Substituting on the RHS of this inequality from (10), and rearranging yields $[(2-\mu) /(1-\mu)] 2 \alpha \theta f^{3}>\gamma^{2}$. Since $(2-\mu) /(1-\mu) \geq 2$, the positive-determinant condition implies satisfaction of this last inequality, yielding the desired conclusion.

${ }^{11}$ For all type- $H$ consumers to undertake air travel while some type- $L$ consumers do not, the inequalities $b^{L}<p+\gamma / f<b^{H}$ must hold. When $\mu=1$, only the second of these inequalities is relevant, and substituting from (12) and (13), it reduces to $\tau+\alpha / 2+\sqrt{2 \gamma \theta}<b^{H}$. Since closed-form $f$ and $p$ solutions are not available when $\mu<1$, the inequalities in $b^{L}<p+\gamma / f<$ $b^{H}$ cannot be rewritten in terms of parameter values in this case. However, when $\mu$ is close to 1 , the $p$ and $f$ solutions will be close to those in (12) and (13). As a result, it follows that, if $b^{L}$ lies well below $\tau+\alpha / 2+\sqrt{2 \gamma \theta}$ while $b^{H}$ lies well above it, the assumed type- $H$ and type- $L$ travel patterns will emerge when $\mu$ is close to 1 .

${ }^{12}$ To see this conclusion, subtract the RHS of (10) from the LHS and differentiate the resulting expression (call it $\Omega$ ) with respect to $\alpha$, which yields $(\gamma / 2) f-(2-\mu) \theta f^{3}$. If this expression is negative or zero, then inspection of (10) shows that $\Omega$ itself must also be negative as long as $\tau>b^{L}$, when in fact $\Omega=0$ must hold at a solution. Therefore, the above derivative must be positive when $\tau>b^{L}$, indicating that $\Omega$ is increasing in $\alpha$. This conclusion in turn 
implies that $f$ is also increasing in $\alpha$ when $\tau>b^{L}$.

${ }^{13}$ Recalling that $\widehat{a}=p+\gamma / f-b^{L}$ and using (14) to eliminate $p$, this requirement translates into the inequality $\left[(1-\mu) \alpha \gamma / 2+\gamma\left(b^{L}-\tau\right)\right] f-\gamma^{2}>0$. Note that while the LHS of condition (10) must be positive at a solution, the latter condition is more stringent. In other words, its satisfaction implies positivity of the LHS of (10), but not vice versa.

${ }^{14}$ The term in brackets equals average type- $L$ loyalty benefits, $2 \int_{a^{*}}^{\alpha / 2}(a / \alpha) d a$.

${ }^{15}$ Heimer and Shy (2006) analyze the sequential case.

${ }^{16}$ When $\mu=0$, the airlines are pure monopolists, which means that sequential and simultaneous choices of $p$ and $f$ must give the same solutions. 